\title{
Tunable Bending Stiffness, Buckling Force, and Natural Frequency of Nanowires and Nanoplates
}

\author{
Hanxing Zhu ${ }^{{ }^{*}}$, Zuobin Wang ${ }^{2}$, Tongxiang Fan ${ }^{3}$, Di Zhang ${ }^{3}$ \\ ${ }^{1}$ School of Engineering, Cardiff University, Cardiff, UK \\ ${ }^{2}$ CNM \& IJRCNB Centers, Changchun University of Science and Technology, Changchun, China \\ ${ }^{3}$ State Key Lab of Metal Matrix Composites, Shanghai Jiao Tong University, Shanghai, China \\ Email: "zhuh3@cf.ac.uk
}

Received June 10, 2012; revised July 8, 2012; accepted August 20, 2012

\begin{abstract}
This paper aims to obtain the simple closed-form results for the combined effects of surface elasticity, initial stress/ strain, and material Poisson ratio on the bending stiffness, natural frequency and buckling force of nanowires and nanoplates. The results demonstrate that all these properties of nanowires or nanoplates can be designed either very sensitive or not sensitive at all to the amplitude of an applied electric potential; show how much of those properties can be controlled to vary; and thus provide a reliable guide to the measurement of the Young's modulus of nanowires/nanoplates and to the design of nano-devices, such as nano-sensors or the cantilever of an AFM.
\end{abstract}

Keywords: Nanowires; Nanoplates; Bending Stiffness; Buckling Force; Natural Frequency

\section{Introduction}

Owing to the large surface area to volume ratio at the nanoscale, the mechanical properties, such as the bending stiffness [1-7], yield strength [8], resonant frequency [9-17] and buckling force [18-21], of nanowires (NWs) and nanoplates (NPs) are size-dependent. In order to interpret the size-dependent mechanical behaviours of NWs and NPs, to extract the mechanical properties (e.g. the Young's modulus) of the material from experimentally measured results, and to design nanoelectro-mechanical systems (NEMS) [22,23], one has to employ a mechanical model and the associated theoretical formula which relates all the parameters involved such as forces/stresses and dimensions. Many theoretical models have been proposed for the purpose for extracting, interpreting, or predicting the Young's modulus [2-7], yield strength [8], resonant frequency [9-17], and buckling force [18-21] of nanowires. However, if the employed theoretical model is incorrect (e.g. [21]), it could mislead our under-standing of the experimentally measured results or result in wrong predictions for the mechanical behaviour of materials/structures or NEMS designed. It has generally been recognised that the initial surface stress can greatly affect the mechanical properties of nanostructures/materials [10-11,14,24-25]. More important and interesting is that the initial surface stress can be controlled to vary

${ }^{*}$ Corresponding author. by adjusting the amplitude of an applied electric potential [17,26-31]. For example, the initial surface stress of $\mathrm{Au}$ (111) is $1.13 \mathrm{~N} / \mathrm{m}$. Biener et al. [26], however, have experimentally found that for nanoporous Au material, by controlling the chemical energy, the adsorbate-induced initial surface stress $\tau_{0}$ can reach $17-26 \mathrm{~N} / \mathrm{m}$. Weissmuller et al. [29, 30] have experimentally demonstrated the recoverable deformation by adjusting amplitude of the initial surface stress via controlling an applied electric potential. There is a linear correlation between surface stress and surface charge in anion adsorption on $\mathrm{Au}(111)$ [31].

This paper aims to provide the precise theoretical results of the combined effects of surface elasticity, initial stress/strain and material Poisson ratio on the bending stiffness, natural frequency and buckling force of nanowires and nanoplates, to give the upper and lower bounds of those tunable properties, to serve as a guide for the design and experimental measurement of nanostructures, and to clarify some existing mistakes in the treatment of the initial surface stresses.

\section{Tunable Bending Stiffness}

The combined effects of the surface elasticity and initial stress/strain on the bending stiffness have been obtained as

$$
D_{b}=\frac{E \pi d^{4}}{64}\left(1+\frac{8 l_{n}}{d}+\frac{v \sigma_{0}^{\chi}}{E}\right)=\frac{E \pi d^{4}}{64}\left(1+\frac{8 l_{n}}{d}+\frac{v \varepsilon_{0}^{x}}{1-v}\right)
$$


for nanowires with a circular cross-section [24],

$$
\begin{aligned}
D_{b} & =\frac{E d^{4}}{12}\left(1+\frac{8 l_{n}}{d}+\frac{3 v \sigma_{0}^{x}}{2 E}\right) \\
& =\frac{E d^{4}}{12}\left(1+\frac{8 l_{n}}{d}+\frac{3 v \varepsilon_{0}^{x}}{2(1-v)}\right)
\end{aligned}
$$

or

$$
\begin{aligned}
D_{b} & =\frac{E d^{4}}{12}\left(1+\frac{4 \sqrt{2} l_{n}}{d}+\frac{v \sigma_{0}^{x}}{E}\right) \\
& =\frac{E d^{4}}{12}\left(1+\frac{4 \sqrt{2} l_{n}}{d}+\frac{v \varepsilon_{0}^{x}}{1-v}\right)
\end{aligned}
$$

for nanowires with a square cross-section [25] when bending is about a neutral plane parallel to the surface plane (1b) or about a diagonal plane (1c). Where, $d$ is the cross-sectional diameter in Equation (1a) or the side length of the square cross-section in Equations (1b) and (1c); $E$ is the Young's modulus of the bulk material; $l_{n}=S / E$ is the intrinsic length of the material at the nano scale; and $S$ is the surface elasticity modulus. In order to simplify the analysis and results, both the surface and the bulk materials are assumed to be isotropic and to have the same Poisson ratio $v$; the initial surface stresses in both the axial and the circular directions are assumed to be the same as $\tau_{0}$. The initial residual stress of the bulk material in the axial direction of the nanowire with either a circular or a square cross-section is thus $\sigma_{0}^{x}=-4 \tau_{0} / d$ because equilibrium in the axial direction has to be held for a free nanowire such as a cantilever. For a uniform nanowire with either a circular or a square cross-section, the initial stresses in the bulk material in both the radial and the circular directions are half of that in the axial direction, i.e. $\sigma_{0}^{r}=\sigma_{0}^{\theta}=\sigma_{0}^{x} / 2=-2 \tau_{0} / d$. The initial residual elastic strain in the axial direction, $\varepsilon_{0}^{x}$, of a nanowire is related to the initial surface stresses by $\varepsilon_{0}^{x}=-4 \tau_{0}(1-v) /(E d)$. The von Mises stress of the bulk material should not exceed the material yield strength $\sigma_{y}$, otherwise, the nanowire would undergo permanent plastic deformation. Atomistic simulation [32] has shown that if the diameter of a gold wire is sufficiently small, it can automatically undergo plastic deformation solely owing to the presence of the initial surface stresses. It is well known that the yield strength, $\sigma_{y}$, of some conductive polymer materials or nano-sized metallic materials can be $0.1 E$ ( $E$ is the Young's modulus) or larger [33]. Biener et al. [26] have experimentally found that for nanoporous Au material, by controlling the chemical energy, the adsorbate-induced surface stress $\tau_{0}$ can reach $17-26 \mathrm{~N} / \mathrm{m}$. If the diameter of the ligaments is $5 \mathrm{~nm}, \sigma_{0}^{x}$ would be $20 \mathrm{GPa}$. As the bulk material discussed in this paper can be either metallic, or polymeric or biological, without losing generality, the von Mises yield strength is assumed to be $\sigma_{y}=0.1 E$. If the actual yield strength of the bulk material of a nanowire/nanoplate is larger or smaller than $0.1 E$, its tunable ranges of the bending stiffness, natural frequency, and axial compressive buckling force can still be obtained by scaling up or scaling down the results that are presented in sections that follow.

According to the aforementioned assumptions, for recoverable elastic deformation, the amplitude of the von Mises stress in the bulk material of a nanowire is limited by $\sigma_{e}=\left|\sigma_{0}^{x} / 2\right| \leq \sigma_{y}=0.1 E$. The corresponding initial strain in the axial direction is $\varepsilon_{0}^{x}=\sigma_{0}^{x}(1-v) / E$. The strain in the radial direction of the nanowire cross-section is related to the initial surface stresses or the initial residual elastic strain in the axial direction by

$$
\varepsilon_{0}^{r}=-\frac{2 \tau_{0}}{E d}(1-3 v)=\frac{\sigma_{0}^{x}}{2 E}(1-3 v)=\frac{1-3 v}{2(1-v)} \varepsilon_{0}^{x}
$$

when the effects of both the surface elasticity and the initial surface stress $\tau_{0}$ are absent (i.e. $S=l_{n}=0$ and $\tau_{0}=\sigma_{0}^{x}=\varepsilon_{0}^{x}=\varepsilon_{0}^{r}=0$ ), the diameter or side-length of the cross-section of a nanowire is assumed to be $d_{0}$ and the corresponding conventional bending stiffness to be $D_{0}=\pi d_{0}^{4} E / 64$ for a circular cross-section or $D_{0}=d_{0}^{4} E / 12$ for a square cross-section. When the effect of the initial surface stresses $\tau_{0}$ is present,

$$
d=d_{0}\left(1+\varepsilon_{0}^{r}\right)=d_{0}\left(1+\frac{1-3 v}{2(1-v)} \varepsilon_{0}^{x}\right)
$$

Substituting Equation (3) into (1a), the combined effects of the surface elasticity and initial stresses on the bending stiffness of a nanowire with a circular crosssection can be obtained as

$$
\frac{D_{b}}{D_{0}}=\left(1+\frac{8 l_{n}}{d}+\frac{v \varepsilon_{0}^{x}}{1-v}\right)\left[1+\frac{1-3 v}{2(1-v)} \varepsilon_{0}^{x}\right]^{4}
$$

when the effect of the surface modulus $S$ is absent (i.e. $l_{n} / d_{0}=0$ or $d_{0} \gg l_{n}$ ), the effect of the initial stress $\sigma_{0}^{x}$ on the dimensionless bending stiffness $D_{b} / D_{0}$ of a nanowire with a circular cross-section is plotted against the possible value of the Poisson ratio $v$ of the bulk material for different amplitudes of the initial stress: $\sigma_{0}^{x}=0.2 E, 0.1 E, 0,-0.1 E,-0.2 E$ (note that the corresponding von Mises stresses are $\sigma_{e}=0.1 E, 0.05 E, 0$, $0.05 E$ and $0.1 E$, respectively), as shown in Figure 1(a). As can be seen from Figure 1(a), when the Poisson ratio of the bulk material $v$ is close to 0 , the normalised bending stiffness, $D_{b} / D_{0}$, of a nanowire can be controlled to vary over a range from 0.65 to 1.4 by adjusting the amplitude of the initial stress $\sigma_{0}^{x}$ from $-0.2 E$ to $0.2 E$ (this, in turn, can be realised by adjusting the amplitude of an applied electric potential). The tunable range of $D_{b} / D_{0}$ 
depends strongly on the Poisson ratio $v$ of the bulk material: approximately proportional to the amplitude of the initial stress $\sigma_{0}^{x}$; narrowing with the increase of $v$; and vanishing when $v \approx 0.4$. When $v$ is larger than 0.4 , the trend of the effect of $\sigma_{0}^{x}$ on $D_{b} / D_{0}$ is reversed. When the effect of the surface elasticity $S$ is present and when $D_{b} / D_{0}$ is fixed at 0.1 , the combined effects of the initial stress $\sigma_{0}^{x}$ and the surface elasticity on the normalised bending stiffness $D_{b} / D_{0}$ of a nanowire with a circular cross-section are plotted against the possible value of the Poisson ratio of the bulk material for different amplitudes of the initial stress: $\sigma_{0}^{x}=0.2 E, 0.1 E, 0$, $-0.1 E,-0.2 E$, as shown in Figure 1(b). When $v$ is very small, $D_{b} / D_{0}$ can be controlled to vary over a range from 1.25 to 2.45 . The larger the amplitude of the initial stress $\sigma_{0}^{x}$, the larger the tunable range of $D_{b} / D_{0}$. The effect of the initial stress $\sigma_{0}^{x}$ on $D_{b} / D_{0}$ reduces with the increase of $v$ and vanishes when $v \approx 0.38$. When $v$ is larger than 0.38 , the trend of the effect is reversed. When the initial stress $\sigma_{0}^{x}=0, D_{b} / D_{0}=1+8 l_{n} / d_{0}=1.8$.

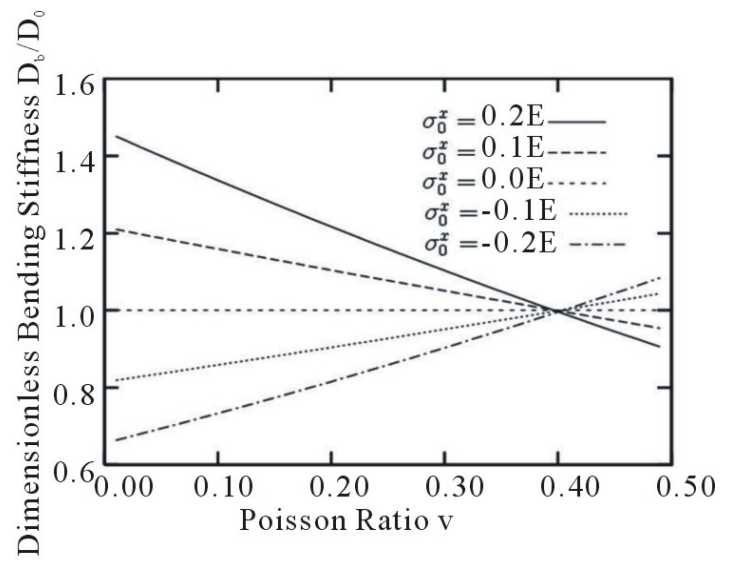

(a)

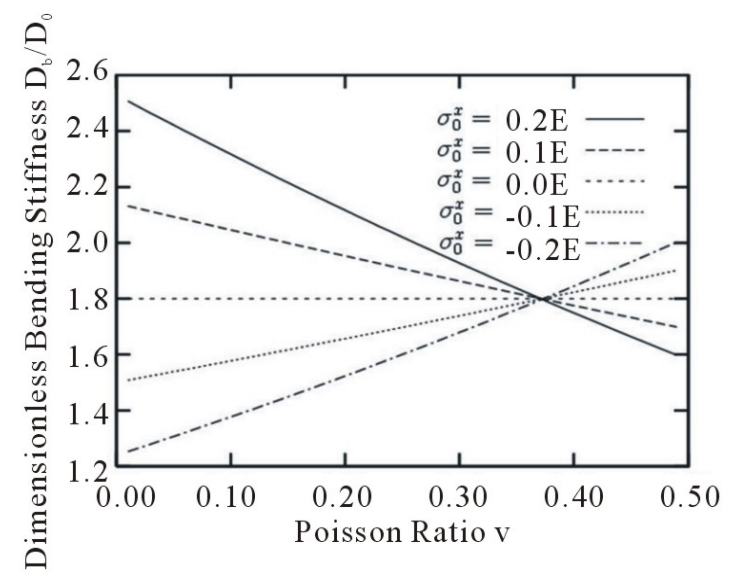

(b)

Figure 1. Effects of the surface elasticity and initial stresses on the bending stiffness of nanowires with a circular cross-section: a) $I_{n} / d_{0}=0$; b) $I_{n} / d_{0}=0.1$.
The relationships between $D_{b} / D_{0}$ and $v$, shown in Figures 1(a) and (b), can well be approximated by the same linear function

$$
\frac{D_{b}}{D_{0}}=1+4 \cdot \frac{l_{n}}{d_{0}}\left[2+(3-9 v) \frac{\sigma_{0}^{x}}{E}\right]+(2-5 v) \frac{\sigma_{0}^{x}}{E}
$$

As the amplitude of the initial stress $\sigma_{0}^{x}=-4 \tau_{0} / d_{0}$ can be controlled to vary by adjusting the amplitude of an applied electric potential [26-31], the normalised bending stiffness of a nanowire $D_{b} / D_{0}$ could be con-trolled to either reduce $30 \%$ or to increase $40 \%$ form the amplitude $1+8 l_{n} / d_{0}$, depending upon the value of the Poisson ratio $v$ of the bulk material. It is noted that for different bulk material, such as a metal, polymer or biological material, the nano-size scale intrinsic length $l_{n}$ may vary between 0.01 to $1 \mathrm{~nm}$. In Figure 1(b), $l_{n} / d_{0}$ is fixed at 0.1 . If $D_{b} / D_{0}$ shown in Figure 1(b) is divided by $\left(1+8 l_{n} / d_{0}\right)=1.8$, the results will be very close to those given in Figure 1(a). This implies that whether the effect of the surface elasticity (i.e. $S$ or $l_{n}$ ) is present or absent, the relative tunable range, $\left(D_{b} / D_{0}\right) /\left(1+8 l_{n} / d_{0}\right)$, of the bending stiffness of a nanowire depends mainly on the amplitude $\sigma_{0}^{x}$ and the bulk material Poisson ratio $v$. Likewise, the bending stiffness of a nanowire with a square cross-section about a diagonal plane or a plane parallel to the surface plane can also be controlled to vary over a large range, depending on amplitude of the initial stress/strain and the material Poisson ratio. The tunable range,

$\left(D_{b} / D_{0}\right) /\left(1+8 l_{n} / d_{0}\right)$, is close to that of a nanowire with a circular cross-section. It is noted that in all the cases, the amplitude of $\sigma_{0}^{x}$ (or $\tau_{0}$ ) is adjustable and controllable, while the Poisson ratio $v$ remains constant for a given material.

For a flat, wide and uniform nanoplate of width $b$ and thickness $h$, the analytical result for the combined effects of the surface elasticity and initial stresses on the bending stiffness is obtained as [25]

$$
D_{b}=\frac{E b h^{3}}{12\left(1-v^{2}\right)}\left[1+6 \frac{l_{n}}{h}+\frac{v(1+v)}{1-v} \varepsilon_{0}^{x}\right]
$$

where $b \gg h ; E, l_{n}=S / E, S$ and the initial surface stress $\tau_{0}$ are exactly the same as those for nanowires. Again, both the surface and the bulk material are assumed to be isotropic and to have the same Poisson ratio $v$ for simplicity in the analysis and results. For recoverable elastic deformation, the initial von Mises stress should not exceed the yield strength of the bulk material, $\sigma_{e}=\left|\sigma_{0}^{x}\right|=\left|\sigma_{0}^{y}\right|=\left|2 \tau_{0} / h\right| \leq \sigma_{y}=0.1 E$. The amplitude of the initial in-plane elastic residual strains of a nanoplate is related to the initial stresses by

$\varepsilon_{0}^{x}=\varepsilon_{0}^{y}=\sigma_{0}^{x}(1-v) / E$. When the initial surface stress $\tau_{0}$ is absent (i.e. $\tau_{0}=\varepsilon_{0}^{x}=\varepsilon_{0}^{y}=\varepsilon_{0}^{h}=0$ ), the width and thickness of the nanoplate are noted as $b_{0}$ and $h_{0}$. 
When the initial surface stress $\tau_{0}$ is present, the strain in the thickness direction of a nanoplate is related to the initial stresses by

$$
\varepsilon_{0}^{h}=\frac{\Delta h}{h_{0}}=\frac{4 v \tau_{0}}{E h}=-\frac{2 v}{E} \sigma_{0}^{x}
$$

and the plate current thickness thus becomes

$$
h=h_{0}\left(1+\varepsilon_{0}^{h}\right)
$$

For simplicity, the initial width $b_{0}$ of the nanoplate is assumed to be a unit and much larger than $h_{0}$ (i.e. $b_{0}=1 \gg h_{0}$ ). Substituting Equations (7) and (8) into (6), the combined effects of surface elasticity, the initial stresses and the Poisson ratio on the bending stiffness of a nanoplate can be obtained and given as

$$
\frac{D_{b}}{D_{0}}=\left[1+6 \frac{l_{n}}{h}+\frac{v(1+v)}{1-v} \varepsilon_{0}^{x}\right]\left(1-\frac{2 v}{1-v} \varepsilon_{0}^{x}\right)^{3}\left(1+\varepsilon_{0}^{x}\right)
$$

where $D_{0}=E h_{0}^{3} /\left(12\left(1-v^{2}\right)\right)$ is the conventional bending stiffness of a nanoplate with a unit initial width when the effects of both the surface elasticity and the initial stress/strain are absent. When the effect of the initial stress is present, the initial unit width becomes $1+\varepsilon_{0}^{y}=1+\varepsilon_{0}^{x}$. That is why this factor appears in Equation (9).

When the effect of the surface modulus $S$ is absent (i.e. $l_{n}=S / E=0$ or $h \gg l_{n}$ ), the effect of the initial stress $\sigma_{0}^{x}$ on the normalised bending stiffness (i.e. $D_{b} / D_{0}$ ) of a nanoplate is plotted against the material Poisson ratio for initial stress fixed at different values: $\sigma_{0}^{x}=-0.1 E$, $-0.05 E, 0,0.05 E$ and $0.1 E$, as shown in Figure 2(a). When the effect of the surface elasticity is present with $l_{n} / h_{0}=0.1$, the similar effect is plotted in Figure 2(b).

As can be seen from Figures 2(a) and (b), the relationship between $D_{b} / D_{0}$ and $v$ can be controlled to vary over ranges from 0.85 to 1.15 when $l_{n} / h_{0}=0$ and from 1.35 to 1.85 when $l_{n} / h_{0}=0.1$, depending strongly on the value of the bulk material Poisson ratio $v$. The relationship given by Equation (9) can be approximated by

$$
\frac{D_{b}}{D_{0}}=1+\frac{1-6 v+v^{2}}{E} \sigma_{0}^{x}+\frac{6 l_{n}}{h_{0}}\left(1+\frac{1-5 v}{E} \sigma_{0}^{x}\right)
$$

When the effect of the initial stress/strain is absent, $D_{b} / D_{0}$ reduces to $1+6 l_{n} / h_{0}$. Similar to nanowires, whether the effect of the surface elasticity (i.e. $S$ or $l_{n}$ ) is present or absent, the relative bending stiffness, $\left(D_{b} / D_{0}\right) /\left(1+6 l_{n} / h_{0}\right)$, of a nanoplate can be controlled to either reduce or increase by up to $15 \%$, depending upon the amplitude of the initial stress and the value of the material Poisson ratio.

Some bulk materials may have a negative surface elasticity modulus [1], i.e. $S<0$ or $l_{n}=S / E<0$. As can be seen from Equations (4) and (9), if the value of

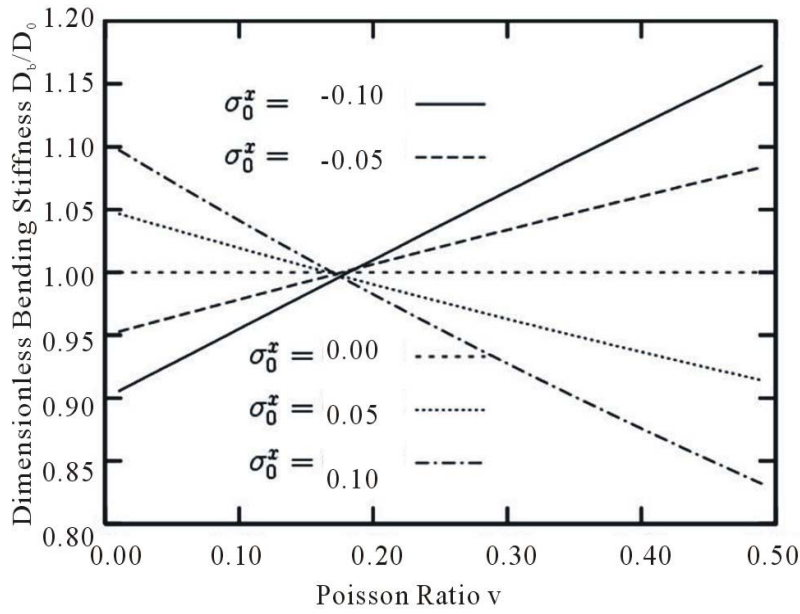

(a)

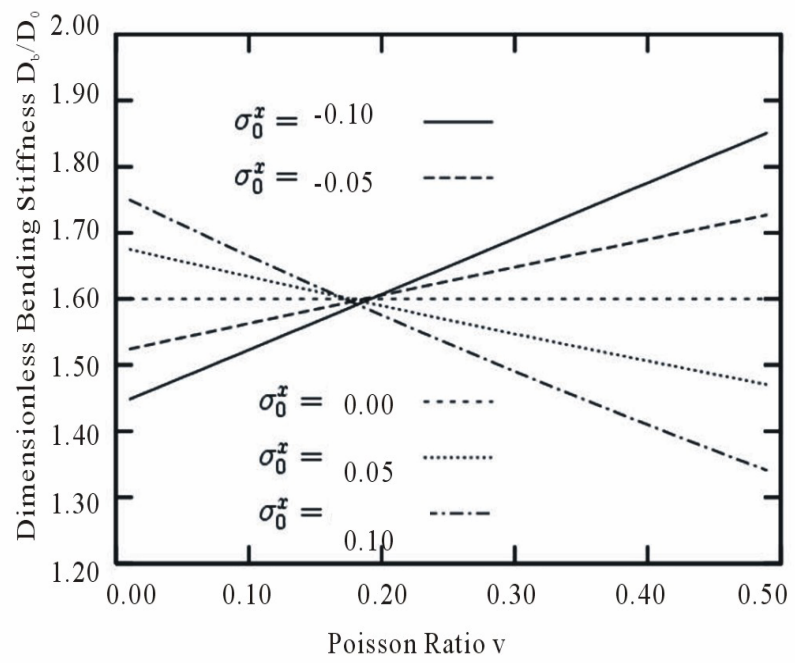

(b)

Figure 2. Effects of the surface elasticity and initial stresses on the bending stiffness of nanoplates: (a) $I_{\mathrm{n}} / h_{0}=0$; (b) $I_{\mathrm{n}} / h_{0}=0.1$.

$8 l_{n} / d_{0}$ (for nanowires) or $6 l_{n} / h_{0}$ (for nanoplates) is close to -1 , by adjusting the amplitude of the initial stress $\sigma_{0}^{x}$ (or $\left.\tau_{0}\right), D_{b} / D_{0}$ could be controlled to vary from the initial positive to subsequent negative. As has been discussed in $[34,35]$, this implies that the nanowire or nanoplate will become unstable and tend to deform automatically into a stable configuration and to output energy at the same time. This can be of useful applications because we sometimes wish a structure or a part to fail or to deform automatically in order to protect others.

\section{Tunable Compressive Buckling Force}

For uniform nanowires or nanoplates with an initial length $L_{0}$, the dimensionless axial compressive buckling force can be obtained as 


$$
\begin{aligned}
& \frac{P_{c r}}{P_{c r}^{0}}=\frac{D_{b} L_{0}^{2}}{D_{0} L^{2}} \\
= & \left(1+\frac{8 l_{n}}{d}+\frac{v \varepsilon_{0}^{x}}{1-v}\right)\left[1+\frac{1-3 v}{2(1-v)} \varepsilon_{0}^{x}\right]^{4} \cdot \frac{1}{\left(1+\varepsilon_{0}^{x}\right)^{2}}
\end{aligned}
$$

for nanowires with a circular cross-section,

$$
\begin{aligned}
\frac{P_{c r}}{P_{c r}^{0}}= & \left(1+\frac{8 l_{n}}{d}+\frac{3 v \varepsilon_{0}^{x}}{2(1-v)}\right) \\
& {\left[1+\frac{1-3 v}{2(1-v)} \varepsilon_{0}^{x}\right]^{4} \cdot \frac{1}{\left(1+\varepsilon_{0}^{x}\right)^{2}} }
\end{aligned}
$$

for nanowires with a square cross-section when buckles about a neutral plane which is parallel to the surface plane,

$$
\begin{aligned}
\frac{P_{c r}}{P_{c r}^{0}}= & \left(1+\frac{4 \sqrt{2} l_{n}}{d}+\frac{v \varepsilon_{0}^{x}}{1-v}\right) \\
& {\left[1+\frac{1-3 v}{2(1-v)} \varepsilon_{0}^{x}\right]^{4} \cdot \frac{1}{\left(1+\varepsilon_{0}^{x}\right)^{2}} }
\end{aligned}
$$

for nanowires with a square cross-section when buckles about a diagonal plane, and

$$
\begin{aligned}
\frac{P_{c r}}{P_{c r}^{0}}= & {\left[1+6 \frac{l_{n}}{h}+\frac{v(1+v)}{1-v} \varepsilon_{0}^{x}\right] } \\
& \left(1-\frac{2 v}{1-v} \varepsilon_{0}^{x}\right)^{3} \cdot \frac{1}{1+\varepsilon_{0}^{x}}
\end{aligned}
$$

for nanoplates. Where $L=L_{0}\left(1+\varepsilon_{0}^{x}\right), P_{c r}^{0}=k \pi^{2} D_{0} / L_{0}^{2}$ is the buckling force of a nanowire or nanoplate when the effects of both the surface elasticity and the initial stress/strain are absent, $P_{c r}=k \pi^{2} D_{b} / L^{2}$, and $k$ is a dimensionless constant depending upon the boundary conditions at the two ends of the nanowire/nanoplate.

For nanowires with a circular cross-section, when the effect of the surface elasticity is absent (i.e. $\left.l_{n} / d_{0}=0\right)$, the effect of the initial stresses on the dimensionless buckling force is plotted in Figure 3(a). When the effect of the surface elasticity is present and $l_{n} / d_{0}=0.1$, the effect of initial stresses on the dimensionless buckling force is presented in Figure 3(b). As can be seen in Figures 3(a) and (b), whether the effect of the surface elasticity is present or absent, the dimensionless buckling force of a nanowire can be controlled to decrease by up to $25 \%$ or to increase by up to $33 \%$, depending strongly on the amplitude of the initial stress and the material Poisson ratio. The larger the material Poisson ratio, the larger is the tunable range of the dimensionless axial compressive buckling force. The trend and amplitudes of the combined effects of the surface elasticity and initial

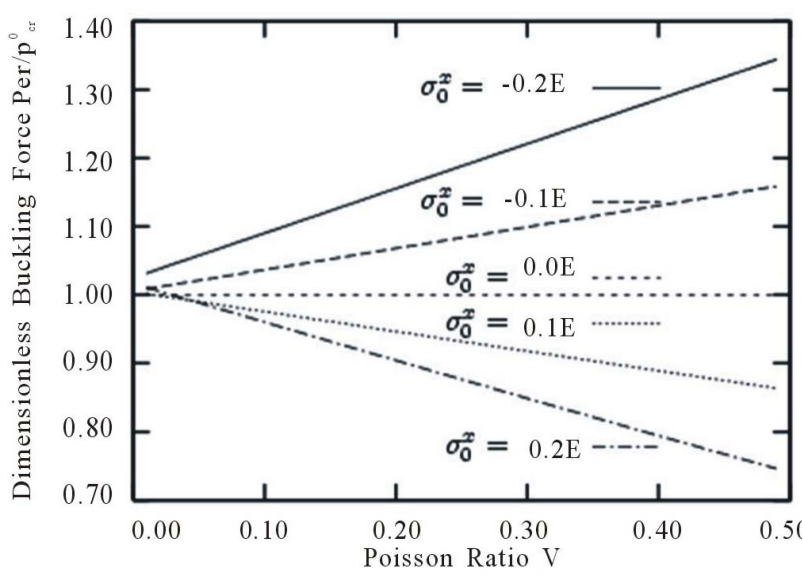

(a)

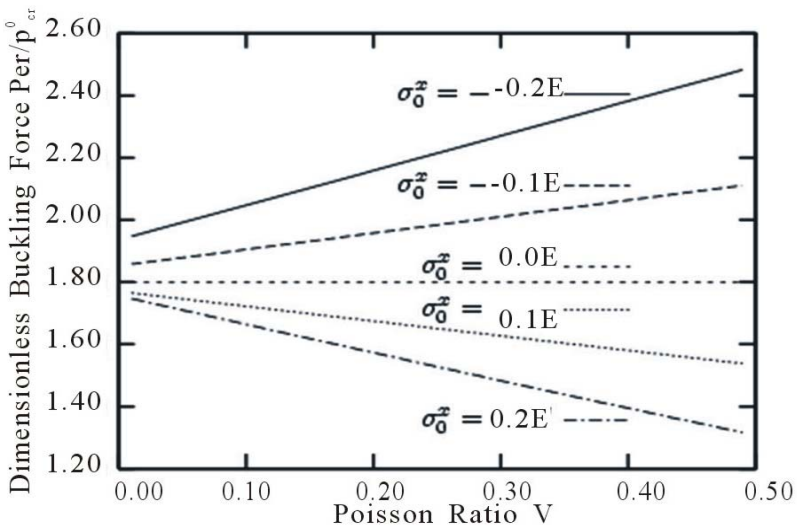

(b)

Figure 3. Effects of the surface elasticity and initial stresses on the dimensionless buckling force of nanowires with a circular cross-section: (a) $I_{n} / h_{0}=0$; (b) $I_{n} / h_{0}=0.1$.

stresses on the dimensionless buckling force of nanowires with a square cross-section, as described in Equations (11b) and (11c), is similar to those given in Figures 3(a) and (b). It should be noted that for a nanowire with a square cross-section, the upper and lower bounds of the tunable dimensionless axial compressive buckling force have to be determined by combining Equations (11b) and (11c) because the nanowire always tends to buckle in the weakest plane. For nanoplates, the combined effects of the surface elasticity and initial stresses on the dimensionless buckling force are presented in Figures 4(a) and (b). As can be seen, whether the effect of the surface elasticity is present or absent, the dimensionless axial compressive buckling force of a nanoplate could be controlled to vary over a range up to $60 \%$, depending strongly on the amplitude of the initial stress and the value of the material Poisson 


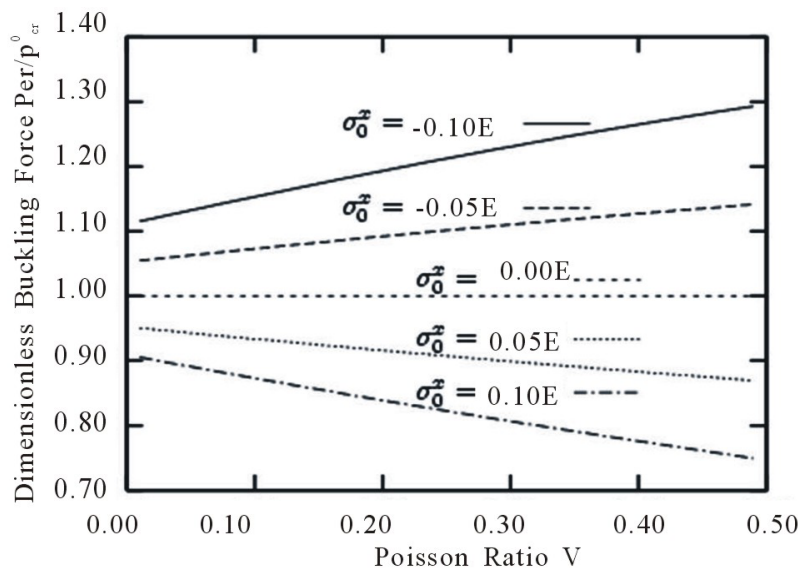

(a)

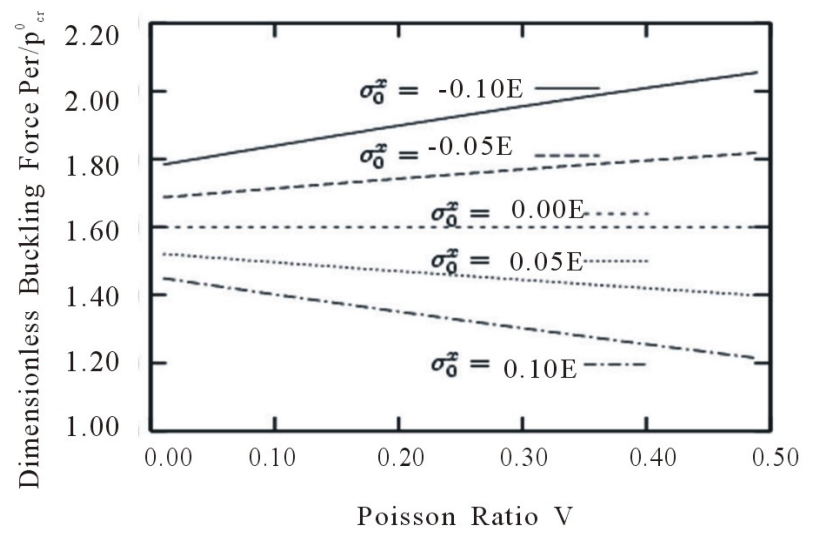

(b)

Figure 4. Effects of the surface elasticity and initial stresses on the dimensionless buckling force of nanoplates: (a) $l_{\mathrm{n}} / h_{0}=0 ;$ (b) $I_{\mathrm{n}} / h_{0}=0.1$.

ratio. It should be noted that the results given in Equations (11) and (12) and shown in Figures 3 and $\mathbf{4}$ apply only when the nanowires/nanoplates are relatively thin and long (e.g. $L / h$ or $L / d \geq 5 \sqrt{E / \sigma_{y}}$ ). Otherwise, the nanowire/nanoplate may yield before losing stability.

Wang and Feng [21] have studied the combined effects of the surface elasticity and initial surface stress on the axial compressive buckling force of nanowires. However, we do not favour their analysis and results. The initial surface stresses are actually internal stresses in a nanowire or nanoplate because the nanowire/nanoplate contains both the bulk and surface materials. If the initial surface stresses are treated as external tractions, their counterparts (i.e. the initial residual stresses in the bulk material) should also be taken into consideration. Wang and Feng [21] only treated the initial surface stresses as external tractions and ignored the effects of their counterpart, and thus obtained the axial compressive buckling force of nanowires as

$$
P_{c r}=k \frac{\pi^{2} E I}{l^{2}}+2 \tau_{0} d
$$

For a very thin and long nanowire (i.e. $l^{2} \gg E I$ ), Equation (13) reduces to $P_{c r}=k \pi^{2} E I / l^{2}+H \approx H=2 \tau_{0} d$ (see the Equation (11) of paper [21]), suggesting that when the initial surface stress $\tau_{0}$ is positive, a very thin and long nanowire will not buckle if the axial compressive force is no larger than $\left|2 \tau_{0} d\right|$. On the other hand, when the initial surface stress $\tau_{0}$ is negative, the bulk material of a nanowire is actually stretched in the longitudinal direction by $\tau_{0}$. Equation (13), however, suggests that a free thin and long cantilever nanowire, which has already stretched in the longitudinal direction by $\tau_{0}$, would buckle even if a tensile force no larger than $\left|2 \tau_{0} d\right|$ is axially applied to stretch it at its two ends. The implications of their results (i.e. (13)) are entirely against the common sense. It is noted that the same treatment (i.e. taking the initial surface stresses as external tractions and ignoring the effects of the initial residual stresses in the bulk materials) has appeared in many research papers on studying the bending stiffness, buckling force and resonant frequency of nanowires, e.g. $[6,11,13,21]$. We do not agree with the way of their treatment on the initial surface stress and thus suspect their obtained results.

\section{Tunable Natural Frequency}

For a uniform cantilever nanowire or nanoplate, when the effect of the initial stresses is absent (i.e. $\tau_{0}$ or $\sigma_{0}^{x}$ is 0 ), the natural frequency is given by

$$
f_{i}=\frac{k_{n}}{2 \pi} \sqrt{\frac{(E I)_{i}}{m_{i} L_{i}^{4}}}=\frac{k_{n}}{2 \pi} \sqrt{\frac{D_{i}}{m_{i} L_{i}^{4}}}
$$

where, $k_{n}$ is a constant for a given vibration mode, e.g. $k_{n}=3.52$ for mode $1 ; D_{i}=\left(E \pi d_{0}^{4} / 64\right)\left(1+8 l_{n} / d_{0}\right)$ for nanowires with a circular cross-section, or $D_{i}=\left(E d_{0}^{4} / 12\right)\left(1+8 l_{n} / d_{0}\right)$ for nanowires with a square cross-section when bending is around a neutral plane parallel to a surface plane, or

$D_{i}=\left(E d_{0}^{4} / 12\right)\left(1+4 \sqrt{2} l_{n} / d_{0}\right)$ for nanowires with a square cross-section when bending is around a diagonal plane, or $D_{i}=\left(E h_{0}^{3} / 12\right)\left(1+6 l_{n} / h_{0}\right)$ for nanoplates of an initial unit width; $m_{i}$ is the mass of per unit length of the nanowire or nanoplate; and $L_{i}$ is the length of the cantilever when the initial stress is absent.

When the effect of the initial stresses/strains is present, the normalised natural frequency becomes

$$
\frac{f}{f_{i}}=\sqrt{\frac{D_{b} m_{i}}{D_{i} m}}\left(\frac{L_{i}}{L}\right)^{2}=\sqrt{\frac{D_{b}}{D_{i}}} \cdot\left(\frac{1}{1+\varepsilon_{0}^{x}}\right)^{\frac{3}{2}}
$$

where $D_{b}$ is the bending stiffness given by Equation (4) 
for nanowires with a circular cross-section or by Equation (9) for nanoplates; $L=L_{i}\left(1+\varepsilon_{0}^{x}\right)$ is the length of the nanowire or nanoplate axially stretched by the initial surface stresses, and $m_{i} / m=L / L_{i}$ because of the mass conservation.

The effects of the surface elasticity and initial stresses on the normalised natural frequency of a cantilever nanowire with a circular cross-section and $l_{n} / d_{0}=0.1$ and a cantilever nanoplate with $l_{n} / h_{0}=0.1$ are plotted against the Poisson ratio of the bulk material, as shown in Figures 5(a) and (b). We also found that the effect of the amplitude $l_{n} / d_{0}$ on the relationship given by Equation (15) is so small that it can be neglected. As can be seen in Figures 5(a) and (b), by adjusting the amplitude of the initial stresses (this can be realised by adjusting the amplitude of an applied electric potential), the normalised natural frequency $f / f_{i}$ could be controlled either to reduce or to increase by up to around $20 \%$ for nanowires or around $15 \%$ for nanoplates from 1, and the tunable range depends upon the amplitude of the initial

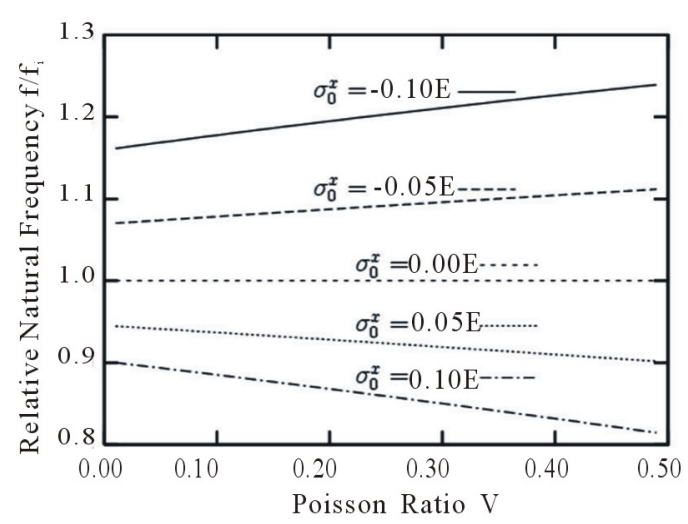

(a)

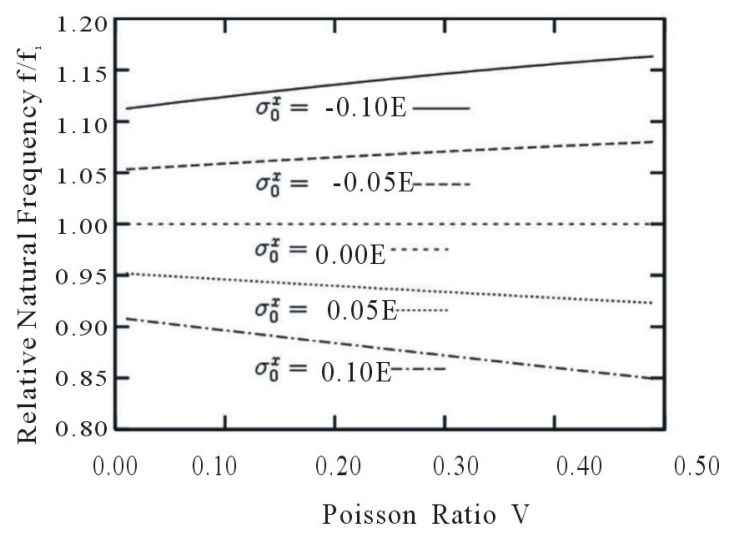

(b)

Figure 5. Effects of the surface elasticity and initials tresses on the normalised natural frequency: (a) For nanowires with a circular cross-section and $l_{n} / h_{0}=0$; (b) For nanoplates with $I_{\mathrm{n}} / h_{0}=0.1$. stresses and the value of the bulk material Poisson ratio. Experiments have demonstrated that micro-or nano-cantilever wires or plates can be used as sensors to monitor the changes in the natural frequency $[17,36]$, and that the elastic properties, such as the Young's modulus, of nanowires can be extracted from the measured natural frequency [37]. Lagowski et al. [16] experimentally found that the normal mode of vibration of thin crystals depends strongly on the surface stress. Wang and Feng [11] and He and Lilley [13] have also studied the resonant frequency of nanowires. As aforementioned, their results are suspicious because they simply treated the initial surface stresses as external tractions and totally ignored the effects of the initial residual stresses in the bulk material.

\section{Conclusion}

The analytic results obtained in this paper demonstrate that the bending stiffness, resonant frequency, and axial compressive buckling force of a nanowire or nanoplate can be designed either very sensitive or not sensitive at all to the amplitude of the initial stresses $\left(\tau_{0}\right.$ or $\sigma_{0}^{x}$ ); show how much those properties can be controlled to vary by adjusting the amplitude of an applied electric potential; and thus provide a reliable guide to the measurement of the Young's modulus of nanowires or nanoplates and to the design of nano-devices, such as nano-sensor or the cantilever of an AFM.

\section{Acknowledgements}

This work is supported by the EC project: PIR-SESGA-2009-247644.

\section{REFERENCES}

[1] R. E. Miller and V. B. Shenoy, "Size-Dependent Elastic Properties of Nanosized Structural Elements," Nanotechnology, Vol. 11, No. 3, 2000, pp. 139-147. doi:10.1088/0957-4484/11/3/301

[2] S. Guenot, C. Fretigny, S. Demoustier-Champagne and B. Nysten, "Surface Tension Effect on the Mechanical Properties of Nanomaterials Measured by Atomic Force Microscopy," Physical Review B, Vol. 69, No. 16, 2004, pp. 165410.1-165410.5. doi:10.1103/PhysRevB.69.165410

[3] J. H. Song, X. D. Wang, E. Riedo and Z. L. Wang, "Elastic Property of Vertically Aligned Nanowires," Nano Letters, Vol. 5, No. 10, 2005, pp. 1954-1958. doi: $10.1021 / \mathrm{n} 1051334 \mathrm{v}$

[4] G. Y. Jing, H. L. Duan, X. M. Sun, Z. S. Zhang, J. Xu, Y. D. Li, J. X. Wang and D. B. Yu, "Surface Effects on Elastic Properties of Silver Nanowires: Contact AtomicForce Microscopy," Physical Review B, Vol. 73, No. 23, 2006, Article ID: 235409. doi:10.1103/PhysRevB.73.235409 
[5] C. Q. Chen, Y. Shi, Y. S. Zhang and J. Zhu, Y. J. Yan, "Size Dependent Young's Modulus in ZnO Nanowires," Physical Review Letters, Vol. 96, No. 7, 2006, Article ID: 075505. doi:10.1103/PhysRevLett.96.075505

[6] J. He and C. M. Lilley, "Surface Effect on the Elastic Behaviour of Static Bending Nanowires," Nano Letters, Vol. 8, No. 7, 2008, pp. 1798-1802. doi: $10.1021 / \mathrm{n} 10733233$

[7] A. Heidelberg, L. T. Ngo, B. Wu, M. A. Phillips, S. Sharma, T. I. Kamins, J. E. Sader and J. J. Boland, "A Generalized Description of the Elastic Properties of Nanowires," Nano Letters, Vol. 6, No. 6, 2006, pp. 11011106. doi: $10.1021 / \mathrm{n} 1060028 \mathrm{u}$

[8] B. Wu, A. Heidelberg and J. J. Boland, "Mechanical Properties of Ultrahigh-Strength Gold Nanowires," $\mathrm{Na}$ ture Materials, Vol. 4, No. 7, 2005, pp. 525-529. doi:10.1038/nmat1403

[9] X. Li, T. Ono, Y. Wang and M. Esashi, "Ultrathin Single-Crystalline-Silicon Cantilever Resonators: Fabrication Technology and Significant Specimen Size Effect on Young's Modulus," Applied Physics Letters, Vol. 83, No. 15, 2003, pp. 3081-3083. doi:10.1063/1.1618369

[10] H. S. Park, "Surface Stress Effects on the Resonant Properties of Silicon Nanowires," Journal of Applied Physics, Vol. 103, No. 12, 2008, Article ID: 123504. doi:10.1063/1.2939576

[11] G. F. Wang and X. Q. Feng, "Effects of Surface Elasticity and Residual Surface Stress on the Natural Frequency of Microbeams," Applied Physics Letters, Vol. 90, No. 23, 2007, Article ID: 231904. doi:10.1063/1.2746950

[12] D. A. Smith, V. C. Holmberg, D. C. Lee and B. A. Korgel, "Young's Modulus and Size-Dependent Mechanical Quality Factor of Nanoelectromechanical Germanium Nanowire Resonators," Journal of Physical Chemistry C, Vol. 112, No. 29, 2008, pp. 10725-10729. doi:10.1021/jp8010487

[13] J. He and C. M. Lilley, "Surface Stress Effect on Bending Resonance of Nanowires with Different Boundary Conditions," Applied Physics Letters, Vol. 93, No. 26, 2008, Article ID: 263108. doi:10.1063/1.3050108

[14] H. S. Park, "Quantifying the Size-Dependent Effect of the Residual Surface Stress on the Resonant Frequencies of Silicon Nanowires if Finite Deformation Kinematics Are Considered," Nanotechnology, Vol. 20, No. 11, 2009, Article ID: 115701 . doi:10.1088/0957

[15] M. E. Gurtin and X. Markenscoff, "Effect of Surface Stress on the Natural Frequency of Thin Crystals," Applied Physics Letters, Vol. 29, No. 9, 1976, pp. 529-530. doi:10.1063/1.89173

[16] J. Lagowski, H. C. Gatos and E. S. Sproles Jr, "Surface Stress and the Normal Mode of Vibration of Thin Crystals: GaAs," Applied Physics Letters, Vol. 26, No. 9, 1975, pp. 493-495. doi:10.1063/1.88231

[17] G. Y. Chen, T. Thundat, E. A. Wachter and R. J. Warmack, "Dsorption-Induced Surface Stress and Its Effects on Resonance Frequencey of Microcantilevers," Journal of Applied Physics, Vol. 77, No. 8, 1995, pp. 3618-3622. doi:10.1063/1.359562

[18] V. V. Dobrokhotov, M. M. Yazdanpanah, S. Pabba, A.
Safir and R. W. Cohn, "Visual Force Sensing with Flexible Nanowires Buckling Springs," Nanotechnology, Vol. 19, No. 3, 2008, Article ID: 035502. doi:10.1088/0957-4484/19/03/035502

[19] C. Hsin, W. Wai, Y. Gu, Y. Gao, C. T. Huang, Y. Liu, L. J. Chen and Z. L. Wang, "Elastic Properties and Buckling of Silicon Nanowires," Advanced Materials, Vol. 20, No. 20, 2008, pp. 3919-3923. doi:10.1002/adma.200800485

[20] M. Riaz, O. Nur, M. Willander and P. Klason, "Buckling of ZnO Nanowires under Uniaxial Compression," Applied Physics Letters, Vol. 92, No. 10, 2008, Article ID: 103118. doi:10.1063/1.2894184

[21] G. F. Wang and X. Q. Feng, "Surface Effects on Buckling of Nanowires under Uniaxial Compression," Applied Physics Letters, Vol. 94, No. 14, 2009, Article ID: 141913. doi:10.1063/1.3117505

[22] F. Xu, Q. Qin, A. Mishra, Y. Gu and Y. Zhu, "Mechanical Properties of $\mathrm{ZnO}$ Nanowires under Different Loading Modes," Nano Research, Vol. 3, No. 4, 2010, pp. 271280. doi:10.1007/s12274-010-1030-4

[23] K. L. Ekinci, M. H. Huang and M. L. Roukes, "Ultrasensitive Nanoelectromechanical Mass Detection," Applied Physics Letters, Vol. 84, No. 22, 2004, pp. 4469-4471. doi:10.1063/1.1755417

[24] H. X. Zhu, "The Effects of Surface and Initial Stresses on the Bending Stiffness of Nanowires," Nanotechnology, Vol. 19, No. 40, 2008, Article ID: 405703. doi:10.1088/0957-4484/19/40/405703

[25] H. X. Zhu, J. Wang and B. L. Karihaloo, "Effects of Surface and Initial Stresses on the Bending Stiffness of Trilayer Plates and Nanofilms," Journal of Mechanics of Materials and Structures, Vol. 4, No. 3, 2009, pp. 589604. doi:10.2140/jomms.2009.4.589

[26] J. Biener, A. Wittstock, L. A. Zepeda-Ruiz, M. M. Biener, V. Zielasek, D. Kramer, R. N. Viswanath, J. Wessmuller, M. Baumer and A. V. Hamza, "Surface-Chemistry-Driven Actuation in Nanoporous Gold," Nature Materials, Vol. 8, No. 1, 2009, pp. 47-51. doi:10.1038/nmat2335

[27] R. Raiteri and H. J. Butt, "Measuring Electrochemically Induced Surface Stress with an Atomic Force Microscope," Journal of Physical Chemistry, Vol. 99, No. 43, 1995, pp. 15728-15732. doi:10.1021/j100043a008

[28] R. C. Cammarata and K. Sieradzki, "Surface and Interface Stresses," Annual Review of Materials Research, Vol. 24, 1994, pp. 215-234. doi:10.1146/annurev.ms.24.080194.001243

[29] J. Weissmuller, R. N. Viswanath, D. Kramer, P. Zimmer, R. Wurschum and H. Gleiter, "Charge-Induced Reversible Strain in a Metal," Science, Vol. 300, No. 5617, 2003, pp. 312-315. doi:10.1126/science. 1081024

[30] D. Kramer, R. N. Viswanath and J. Weissmuller, "Surface-Stress Induced Macroscopic Bending of Nanoporous Gold Cantilevers," Nano Letters, Vol. 4, No. 5, 2004, pp. 793-796. doi:10.1021/n1049927d

[31] W. Haiss, R. J. Nichols, J. K. Sass and K. P. Charle, "Linear Correlation between Surface Stress and Surface Charge in Anion Adsorption on Au(111)," Journal of Electroanalytical Chemistry, Vol. 452, No. 2, 1998, pp. 
199-202. doi:10.1016/S0022-0728(98)00137-5

[32] J. Diao, K. Gall, M. L. Duan and J. A. Zimmerman, "Atomistic Simulations of the Yielding of Gold Nanowires," Acta Materialia, Vol. 54, No. 3, 2006, pp. 643-653. doi:10.1016/j.actamat.2005.10.008

[33] Z. C. Lin and J. C. Huang, "A Study on a Rigid Body Boundary Layer Interface Force Model for Stress Calculation and Stress - Strain Behaviour of Nanoscale Uniaxial Tension," Nanotechnology, Vol. 15, No. 11, 2004, pp. 1509-1518. doi:10.1088/0957-4484/15/11/024

[34] H. X. Zhu, "Size-Dependent Elastic Properties of Microand Nano-Honeycombs," Journal of the Mechanics and Physics of Solids, Vol. 58, No. 5, 2010, pp. 696-709. doi:10.1016/i.jmps.2010.02.009

[35] H. X. Zhu, L. B. Yan, R. Zhang and X. M. Qiu,
"Size-Dependent and Tunable Elastic Properties of Hierarchical Honeycombs with Regular Square and Equilateral Triangular Cells," Acta Materialia, Vol. 60, No. 12, 2012, pp. 4927-4939. doi:10.1016/j.actamat.2012.05.009

[36] V. Tabard-Cossa, M. Godin, L. Y. Beaulieu and P. Grutter, "A Differential Microcantilever-Based System for Measuring Surface Stress Changes Inducted by Electrochemical Rection," Sensors and Actuators B: Chemical, Vol. 107, No. 1, 2005, pp. 233-241.

doi:10.1016/j.snb.2004.10.007

[37] Z. L. Wang, Z. R. Dai, R. P. Gao and J. L. Gole, "Measuring the Young's Modulus of Solid Nanowires by in Situ TEM," Journal of Electron Microscopy, Vol. 51, Suppl. 1, 2002, pp. S79-S85.

doi:10.1093/jmicro/51.Supplement.S79 